\title{
Polarization Phenomena in Periodic Metasurfaces at Oblique Incidence
}

\author{
Sergey S. Kruk, ${ }^{1}$ Alexander Poddubny ${ }^{2}$, Christian Helgert, ${ }^{1,3}$ Manuel Decker, ${ }^{1}$ David A. Powell, ${ }^{1}$ \\ Thomas Pertsch, ${ }^{3}$ Dragomir N. Neshev, ${ }^{1}$ and Yuri S. Kivshar ${ }^{1}$ \\ ${ }^{1}$ Nonlinear Physics Centre and Centre for Ultrahigh Bandwidth Devices for Optical Systems (CUDOS), \\ The Australian National University, Canberra, ACT 0200, Australia \\ ${ }^{2}$ Ioffe Institute and ITMO University, St. Petersburg, Russia \\ ${ }^{3}$ Institute of Applied Physics, Abbe Center of Photonics, \\ Friedrich-Schiller-Universität Jena, 07743 Jena, Germany \\ Dragomir.Neshev@anu.edu.au
}

\begin{abstract}
We study experimentally and theoretically oblique incidence polarization phenomena for periodic metasurfaces of different symmetries. We show that the strength of the optical activity, circular dichroism and asymmetric transmission is dependent on the rotational symmetry of the metasurface lattice. We verify the predictions of the analytical model by the experimental study of optical metasurfaces with 4-fold, 6-fold and 5-fold rotational symmetry and demonstrate an excellent agreement.
\end{abstract}

\section{INTRODUCTION}

Metasurfaces are 2D arrangements of individual sub-wavelength particles made of composite materials. Their design is optimized for a desired operation and functionality with respect to their interaction with electromagnetic radiation. Metasurfaces have recently attracted an attention due to their ability to abruptly change the phase of light, allowing for polarization and wavefront control. Metasurfaces have been recently demonstrated to operate as flat lenses [1], highly-efficient polarization converters [2], vortex beam generators [2], etc. The properties of metasurfaces are defined by the response of an individual particle as well as by its coupling to the neighbors. While significant efforts have been made on engineering individual particles for desired functionality, the ability to tune the properties of metasurfaces by arranging the particles into different layouts remains widely unexplored and unexploited.

Here we highlight the impact of the inner arrangement of elements on metasurfaces' properties. We present an analytical model, which quantitatively describes optical properties of a metasurface depending on the symmetry of its inner arrangement for different illumination conditions, and experimentally demonstrate the polarization phenomena in optical metasurfaces, which are in excellent agreement with the theory.

\section{ANALYTICAL MODEL}

We start the microscopic analytical derivation of the polarization-dependent scattering and transmission from the arbitrary metasurface with the $\mathrm{D}_{N h}$ symmetry. To this end, we consider a discrete electric dipole approximation. The structure is described as an array of identical scatterers with polarizability $\hat{\alpha}$, arranged in the $x y$ plane, centered in the points $\boldsymbol{a}$ and embedded in air. To focus on an impact of the symmetry of the arrangement, we consider an individual scatterer to be cylindrically symmetric, which means that the polarizability tensor is diagonal and $\alpha_{x x}=\alpha_{y y} \equiv \alpha$. For simplicity we also neglect transverse polarizability, $\alpha_{z z}=0$, which is reasonable for thin metasurfaces. In this approximation the structure has the symmetry plane $z=0$. The equation for the electric field reads

$$
\operatorname{rot} \operatorname{rot} \boldsymbol{E}=\left(\frac{\omega}{c}\right)^{2} \boldsymbol{E}+4 \pi \hat{\alpha} \sum_{\boldsymbol{a}} \boldsymbol{E}(\boldsymbol{a}) \delta(\boldsymbol{r}-\boldsymbol{a}) .
$$

The incident wave is characterized by the amplitude $\boldsymbol{E}_{i}$ and the wave vector $\boldsymbol{k}$. In order to determine the scattered waves we expand the electric field over the plane wave harmonics, generalizing the approach of Ref. [3] to the case of oblique incidence. In both periodic and quasicrystalline metasurfaces the Bragg diffraction leads to the polarization-dependent coupling of different plane wavevector harmonics. Since the lattice spacing in the metasurface is larger than the wavelength, the diffraction phenomena can be considered as a perturbation. As a results, we obtain the matrices, characterizing transmitted $(\widehat{\mathrm{T}})$ and specularly reflected $(\widehat{\mathrm{R}})$ waves in the basis of TE and TM polarizations 


$$
\begin{gathered}
\widehat{\mathrm{T}}=\widehat{\mathrm{S}}_{++}=\left(\begin{array}{cc}
1+U & V \cos \theta \sin ^{\widetilde{N}-2} \theta \sin \widetilde{N} \varphi \\
V \cos \theta \sin ^{\widetilde{N}-2} \theta \sin \widetilde{N} \varphi & 1+U \cos ^{2} \theta
\end{array}\right)=\left(\begin{array}{ll}
T_{11} & T_{12} \\
T_{21} & T_{22}
\end{array}\right), \\
\widehat{\mathrm{R}}=\widehat{\mathrm{S}}_{-+}=\left(\begin{array}{cc}
U & -V \cos \theta \sin ^{\widetilde{N}-2} \theta \sin \widetilde{N} \varphi \\
-V \cos \theta \sin ^{\widetilde{N}-2} \theta \sin \widetilde{N} \varphi & U \cos ^{2} \theta
\end{array}\right)=\left(\begin{array}{ll}
R_{11} & R_{12} \\
R_{21} & R_{22}
\end{array}\right),
\end{gathered}
$$

where $\widetilde{N}$ is the order of rotational symmetry of metasurfaces arrangement for even symmetries and doubled order for odd symmetries, $U=i \alpha \omega / c S$ and $V=-\widetilde{N} \omega /(c G)|f|^{2} e^{-(G R)^{2}} U^{\widetilde{N}-2}$. Here $S$ is an area, occupied by an individual particle, $\boldsymbol{G}$ is the characteristic lattice diffraction vector, $f(\boldsymbol{G})=\sum_{a} e^{i G a} / \mathcal{N}$ is the corresponding structure factor $(\mathcal{N}$ is the number of scatterers) and $R$ is a cutoff parameter on the order of the individual scatterer size, specifying the spatial scale when the dipole approximation stops being valid.

Considered $\mathrm{D}_{N h}$ symmetry assumes the metasurface to be embedded into a homogeneous medium. Our results can be easily extended for the metasurface on a homogeneous substrate $\left(\mathrm{C}_{N v}\right.$ symmetry $)$ as follows:

$$
\hat{T}\left(C_{N v}\right)=\left(\begin{array}{cc}
t_{\mathrm{TE}} & 0 \\
0 & t_{\mathrm{TM}}
\end{array}\right) \hat{T}\left(D_{N \mathrm{~h}}\right), \quad \hat{R}\left(C_{N v}\right)=\left(\begin{array}{cc}
r_{\mathrm{TE}} & 0 \\
0 & r_{\mathrm{TM}}
\end{array}\right) \hat{R}\left(D_{N \mathrm{~h}}\right),
$$

where $t_{\mathrm{TE}}, t_{\mathrm{TM}}, r_{\mathrm{TE}}$ and $r_{\mathrm{TM}}$ are the transmission and reflection coefficients from the substrate itself for principal polarizations. We notice that the expressions in circular basis can be obtained from the expressions in linear basis using the basis change operator

$$
\widehat{\Lambda}=\frac{1}{\sqrt{2}}\left(\begin{array}{cc}
1 & 1 \\
i & -i
\end{array}\right) \text {. }
$$

Finally, we note that the relations between the $\widehat{T}$ and $\widehat{\mathrm{R}}$ components and several polarization phenomena: asymmetric transmission relates to $\left|T_{12}\right|^{2}-\left|T_{12}\right|^{2}$ in linear basis, optical activity is Phase $\left(T_{11}{ }^{2}\right)-$ Phase $\left(T_{22}{ }^{2}\right)$ in circular basis, and circular dichroism (CD) is $\left|T_{11}\right|^{2}-\left|T_{22}\right|^{2}$ in circular basis.

\section{EXPERIMENTAL VERIFICATION}

To verify our model experimentally we first focus on a particular polarization phenomenon: circular dichroism for metasurfaces on a substrate. Our analytical model predicts the following expression for the circular dichroism:

$$
\delta_{\text {circ }} \sim \cos \theta \sin ^{\widetilde{N}} \theta \sin \widetilde{N} \varphi,
$$

where $\theta$ and $\varphi$ are the polar and azimuthal angles of illumination. We experimentally verify this expression versus its three parameters: $\widetilde{N}, \theta$ and $\varphi$. For this purpose we fabricate three optical metasurfaces with $N=$ $4,6,5$, and correspondingly $\widetilde{N}=4,6$, and 10 [see Fig. 1 (b-d)]

meta-atom

(a)

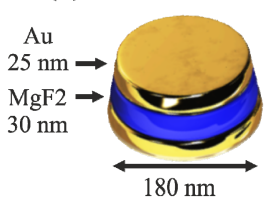

square lattice

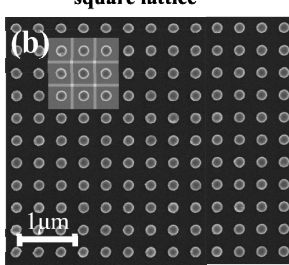

hexagonal lattice

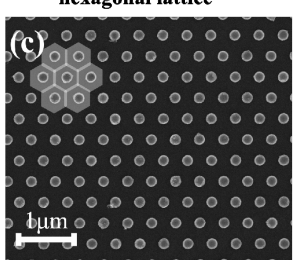

Penrose tiling

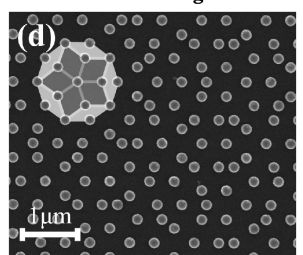

(e)

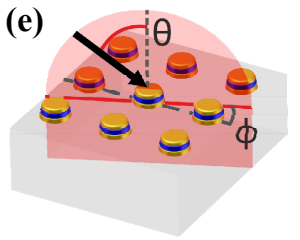

Fig. 1. Metasurfaces. (a) a single nanoparticle, (b-d) scanning electron micrographs of three fabricated metasurfaces with . $\widetilde{N}=4,6$, and 10 respectively, and (e) visualization of oblique illumination of a metasurface with polar angle $\theta$ and azimuthal angle $\varphi$.

The fabrication is done using electron-beam lithography with sequential evaporation of three layers of gold $/ \mathrm{MgF}_{2} /$ gold and a lift-off. The metasurfaces exhibit two resonant responses in spectral region between $0.5 \mu \mathrm{m}$ and $1 \mu \mathrm{m}$ wavelength associated with electric dipole and magnetic dipole excitations, respectively [4].

We use a white-light transmission spectroscopy setup to measure circular dichroism [4] versus the three parameters $\widetilde{N}, \theta$ and $\varphi$ and integrate the circular dichroism over the aforementioned spectral region. The results are summarized in Fig. 2 (a-c). As seen in Fig. 2, the experimental measurements follow well the theoretically predicted trends. 
(a)

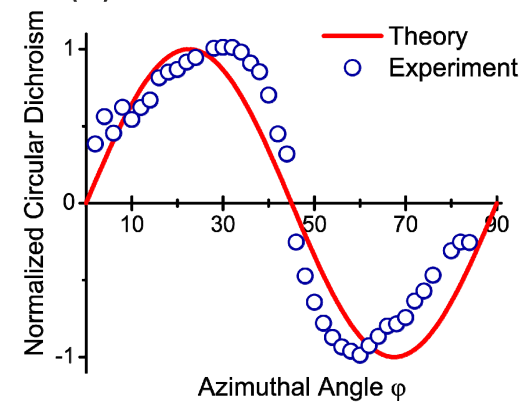

(b)

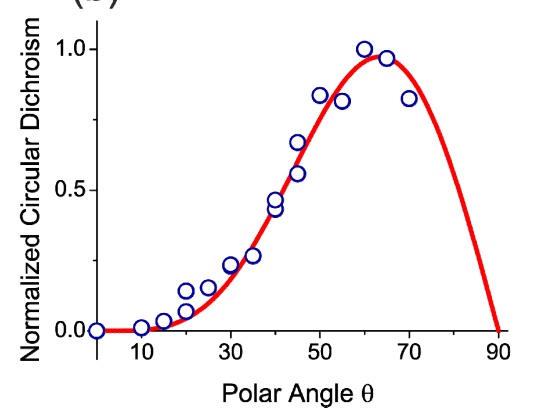

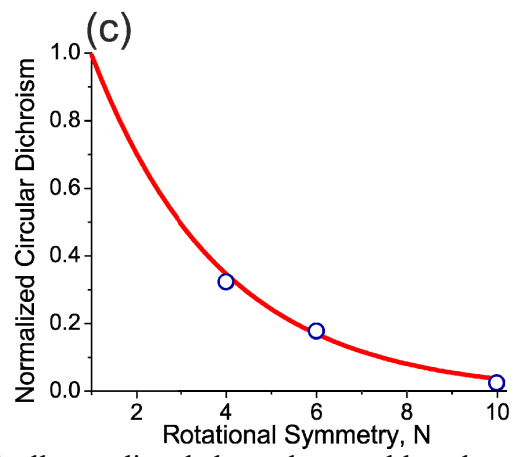

Fig. 2. Comparison of experiment with theory. Red lines correspond to theoretically predicted dependences, blue dots correspond to experimentally measured data. (a) $\mathrm{CD}$ vs $\varphi$ for $\widetilde{N}=4 \& \theta=45^{\circ}$ ). (b) $\mathrm{CD}$ vs $\theta$ for $\widetilde{N}=4 \& \sin (\widetilde{N} \varphi)=1$. (c) $\mathrm{CD}$ vs $\widetilde{N}$ for $\theta=45^{\circ} \& \sin (\widetilde{N} \varphi)=1$

The azimuthal dependence of the dichroism exhibits oscillations. In agreement with the general symmetry analysis, the values of $\delta_{\text {circ }}$ vanish when the light incidence plane coincides with the vertical symmetry plane. Moreover, in case of odd metasurface rotational symmetry in real space $N$, the effective rotational degree $\widetilde{N}$ is twice larger, particularly, for the Penrose tiling in Fig. 1(d) $\widetilde{N}=10$. The dichroism degree also vanishes at both normal $(\theta=0)$ and gliding $(\theta=\pi / 2)$ incidence. It has a maximum at $\theta=\arctan (\sqrt{\widetilde{N}})$, that shifts to larger angles at higher rotational symmetry. The peak values of the dichroism degree decrease with $\widetilde{N}$ as well.

\section{CONCLUSION}

Taking advantage of symmetry considerations, we have analysed the potential of various metasurface arrangements to affect the polarization phenomena upon oblique illumination. The presented methodology that using discrete dipole approximation, provides an unambiguous language for discussing the impact of the inherent symmetry of the metamaterial lattices on their far-field response. Our findings allow the quantification analysis of the impact of inter-element coupling and lattice symmetry on the optical properties of metamaterials, and to separate this contribution from the response associated with a single meta-atom. Our findings constitute an important step towards the design of optically isotropic metamaterials and metasurfaces.

\section{ACKNOWLEDGEMENT}

The authors acknowledge the financial and facility support from the Australian Research Council (ARC), the Federal Ministry of Education and Research (PhoNa, Germany) and the Thuringian State Government (MeMa, Germany). C.H. gratefully acknowledges a postdoctoral fellowship from the German Academic Exchange Service (DAAD). A.P. has been supported by the Russian Foundation for Basic Research and the Grant of the President of the Russian Federation No. MK-6029.2014.2. All authors thank F. Karouta, K. Vora, and J. Tian from the Australian National Fabrication Facility, as well as A. Rose for the useful discussions.

\section{REFERENCES}

[1] F. Aieta et al., "Aberration-Free Ultrathin Flat Lenses and Axicons at Telecom Wavelengths Based on Plasmonic Metasurfaces” Nano Lett. 12, 4932 (2012)

[2] Y. Yang et al., "Dielectric Meta-Reflectarray for Broadband Linear Polarization Conversion and Optical Vortex Generation” Nano Lett. 14, 1394 (2014)

[3] A.N. Poddubny, "Wood anomalies in resonant photonic quasicrystals” Phys. Rev. B 83, 075106 (2011)

[4] S. S. Kruk et al., "Optical metamaterials with quasicrystalline symmetry: Symmetry-induced optical isotropy” Physical Review B 88, 201404(R) (2013). 